\section{Understanding Electrosurgical Unit Perturbations in Order to Address Hospital Operating Room Electromagnetic Compatibility}

\author{
Mireya Fernández-Chimeno*, Marcos Quílez, and Ferran Silva
}

\begin{abstract}
We analyze the radiated emissions from a low power electrosurgical unit (ESU). Measurements at a 3-meter distance are performed in order to find a suitable measurement setup intended to reproduce realistically the real working ESU behavior in a test lab. The broad-band and narrow-band characteristics of the perturbation are studied in order to address the other equipment immunity.
\end{abstract}

Index Terms-Electrosurgical unit EMI measurements, ESU electromagnetic emission measurement setup, operating room electromagnetic compatibility.

\section{INTRODUCTION}

One of the most concerning electromagnetic interference (EMI) sources in a hospital operating theatre is the electrosurgical unit (ESU). ESUs have often been identified as one of the main interference sources in these environments [1], [2] as well as being a direct cause of malfunction of other critical medical equipment [3], [4]. Although recommendations for the preparation of operating theatres mention the danger of the ESU electromagnetic interference, they do not propose any solution [5]. In order to work as intended, an ESU has to generate high power currents (about $700 \mathrm{~mA}$ for a $300-\mathrm{W}$ model) at frequencies of hundreds of kilohertz. ESU cutting working mode is achieved primarily with a continuous sinusoidal waveform, whereas coagulation is achieved primarily with a series of sinusoidal wave packets (a pulsed sinusoidal wave) [6]. This pulsed power signal applied to the patient allows tissue to be cut and to coagulate, but at the same time it generates such high electromagnetic perturbations that surgeons are concerned about their own exposure to them during operations [7]. The ESU interference is low frequency (hundreds of kilohertz) and easily reaches the other electronic devices in the electromagnetic near-field area because of the proximity of this equipment in the operating theatre. Solutions such as "keeping the ESU output as low as possible" or "monitoring the sensitive device operation while using electro surgery" [3] cannot be accepted nowadays in a high-risk scenario like an operating theatre.

In order to determine an EMI measurement method that provides a realistic value of ESU electromagnetic radiated emissions, several measurements have been performed with different load models such as resistors or animal meat. The aim is to obtain a reproducible measurement method that can be performed in the test laboratory and which models the radiated emissions in a situation similar to that found in a hospital. Fig. 1 shows the radiated emissions (magnetic field) from a low-power $(70 \mathrm{~W})$ ESU in two different situations. In both cases, the measurements are performed with an electrically shielded loop antenna

Manuscript received May 17, 2005; revised November 6, 2005. This work was supported in part by the Spanish Ministerio de Educación y Ciencia under the Project Grant DPI2004-07865-C02-01. Asterisk indicates corresponding auhtor.

*M. Fernández-Chimeno is with the Electromagnetic Compatibility Group, Electronic Engineering Department, Technical University of Catalonia, C/Jordi Girona 1-3, Edifici C-4, 08034 Barcelona, Spain (e-mail: mireya@eel.upc.edu).

M. Quílez and F. Silva are with the Electromagnetic Compatibility Group, Electronic Engineering Department, Technical University of Catalonia, 08034 Barcelona, Spain.

Digital Object Identifier 10.1109/TBME.2006.873389
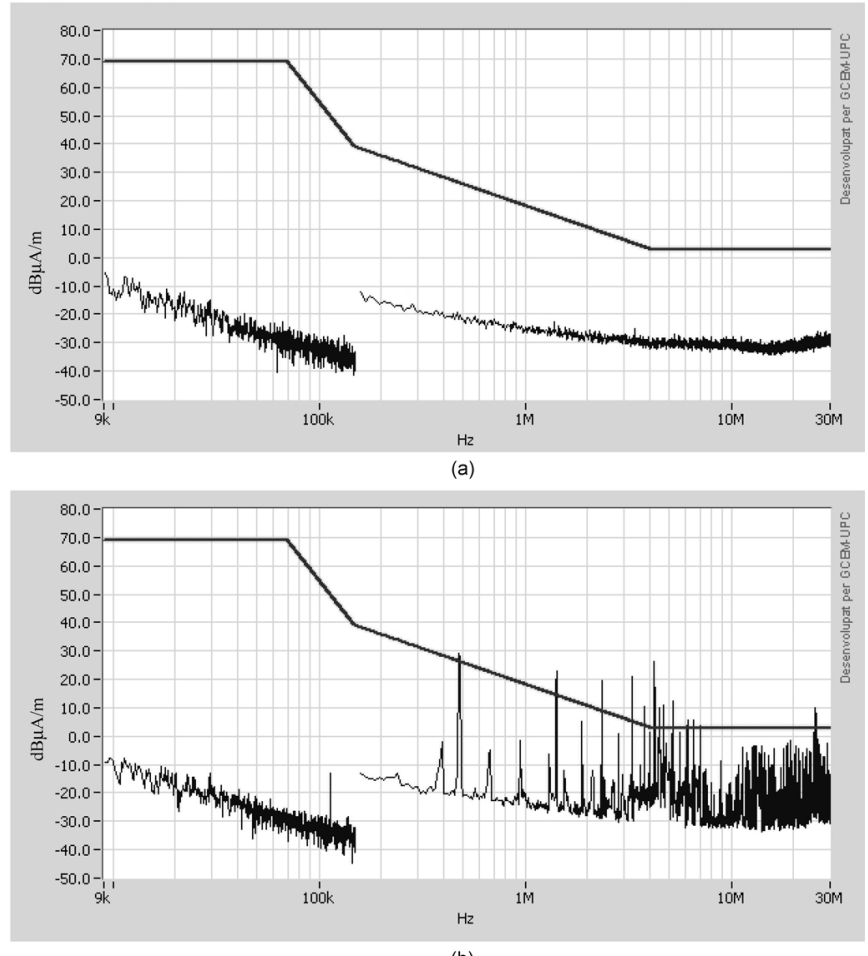

(b)

Fig. 1. Radiated EMI from a 70-W ESU measured with a loop antenna at 3-m distance showing the standard limits. (a) ESU in standby following IEC 60601-2-2. (b) ESU cutting a piece of meat.

at 3-meter (m) distance and with the 9-kHz CISPR resolution bandwidth. In the first situation [Fig. 1(a)], the device is tested according the standard IEC $60601-2-2$ [8]; that is, with the device connected but in its standby mode. In the second situation [Fig. 1(b)], the measurement is performed using the device to cut a piece of meat. It is clear that this device (and perhaps all ESU models) fail to comply with the prevailing standard when they are working as intended. Furthermore, the objective of this EMC standard emissions limit is to prevent malfunctions of communications systems located far away while the main risk in the operating room is the malfunction of vital electronic systems working inside the same room. Therefore, the characteristics of the interference and its effect must be analyzed in order to redefine the limit or the measurement procedure.

The situation of the ESU in a medical environment (for example an operating room) is a near field situation but, it is difficult nowadays to implement an easily reproducible EMC test method based on near-field measurements [9]. Therefore, this paper seeks to define the conditions and the setup for the measurements that give realistic information by measuring at a distance of 10 or $3 \mathrm{~m}$ with the usual EMC antennas and methods. The equipment has to work in the EMC test lab in a similar way to the operating room in the hospital. This is not easy if we consider that its actual use in a hospital it is to cut biological tissue from a patient and what is required is a method that can easily be reproduced in the test lab. Several measurements have been performed with the aim of obtaining such a method.

\section{RADIATED EMISSIONS FROM AN ESU}

The first measurements were taken to compare the emissions in the standby mode (as specified in the standard) and while working in a real cutting mode. Now, a resistor is used as a load to model the patient 
as it is done in some EMC laboratories. The resistor was chosen to obtain the maximum power transfer and the output power was set at $95 \%$. Both measurements are performed at $3 \mathrm{~m}$ in the frequency range of 1-10 MHz, using a shielded loop antenna for the $\mathrm{H}$ field and a rod antenna for the E field measurements. The frequency band is chosen because it appears to have more frequency content than the other ones (see Fig. 1).

Although the measurements with a resistor as a load are closer to the way an ESU actually works, other factors must be considered in the measurement setup since they can have an influence on the equipment emissions. The first point to consider is the presence of an operator (the surgeon); that is, a conductive body placed near the ESU when it is working. We have measured the ESU emissions both with and without a volunteer holding the active electrode wire. The ESU was loaded with a resistor and the power set at $95 \%$ of its maximum. Differences of up to $8 \mathrm{~dB}$ in the spectrum peaks were found due to the presence of an operator in the measurement setup, so it is important to take such an operator into account in the radiated emission test.

The second point to analyze is whether a resistor is a good load model for a biological tissue when the ESU radiated emissions are measured. To know how good a model a resistor is, several measurements have been performed when cutting a piece of meat ( $1 \mathrm{~kg}$ of pork loin). The radiated EMI when cutting meat is $40 \mathrm{~dB}$ larger than when a resistor is used as a load. Fig. 2 summarizes the measurement results for the resistor load and cutting meat situations.

The increase in the emission level when cutting meat may be due to two factors: first, the impedance of a biological tissue may not be resistive at the ESU working frequencies; second, when cutting the piece of meat, electrical arcs appear between the tissue and the tip of the active electrode (some ESU working modes require the presence of arcing), so the increase in the emission levels could also be attributable to arcing. Concerning the first hypothesis, the ESU working frequencies are usually in the hundreds of kilohertz band and there are harmonics up to several tens of megahertz. However the muscular tissue impedance can be considered resistive in the measurement bandwidth (1-10 MHz.) [10], [11], so the increase in the emission level must be attributed to the arcing phenomenon.

At this point it is necessary to measure the radiated emission of an ESU when arcing, by using a more repeatable measurement setup than a piece of meat. Some life supporting medical devices standards like EN60601-2-25 [12] specify a measurement setup for testing the immunity against ESU interferences. We propose a measurement setup (shown in Fig. 3) based on that specified by the EN60601-2-25 standard. The emission measurement starts with the active electrode (B) in contact with the metal plate (C). Then the active electrode is slowly moved away a short distance from the metal plate to start and maintain arcing. Fig. 2 shows the measurement results together with the load resistor and cutting meat measurements. Values of $0.5 \mathrm{~V} / \mathrm{m}$ were recorded at a distance of $3 \mathrm{~m}$.

The increase in the emission peaks during arcing suggests that broadband noise is present. To verify this hypothesis, we repeat the measurements with a narrower resolution bandwidth of $10 \mathrm{~Hz}$, centered on the first ESU harmonic at $1.4 \mathrm{MHz}$. Fig. 4 shows the results for $\mathrm{H}$ field. In this case, the interference peaks have the same level which confirms the broad-band nature of the noise generated when arcing.

At this point, a look at the time domain current signal can improve knowledge about this interference. LaCourse et al. measured in [13] the voltage-current characteristic of an electrosurgical arc. Their measurements show a sinusoidal behavior when there is no arcing and a repetitive transient behavior when the arc is generated. This transient behavior could explain the radiated broad-band spectrum measured when there is arcing. We have measured the electric field at $3 \mathrm{~m}$. in the time domain using a rod antenna and an oscilloscope, in the following two

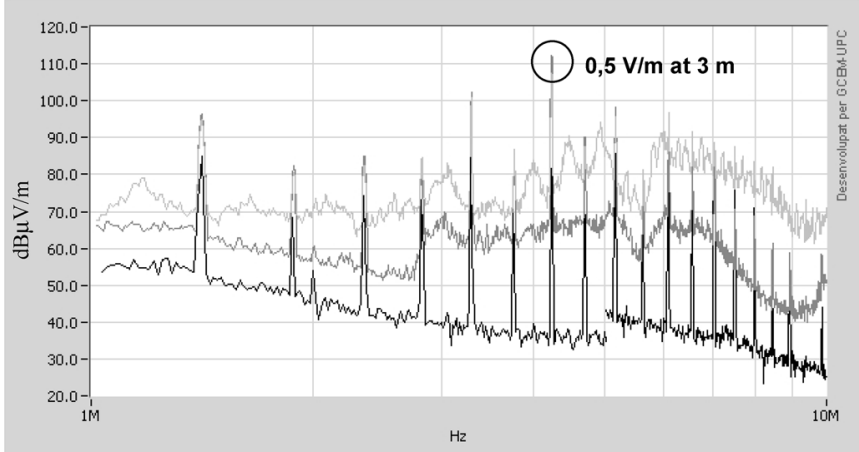

(a)

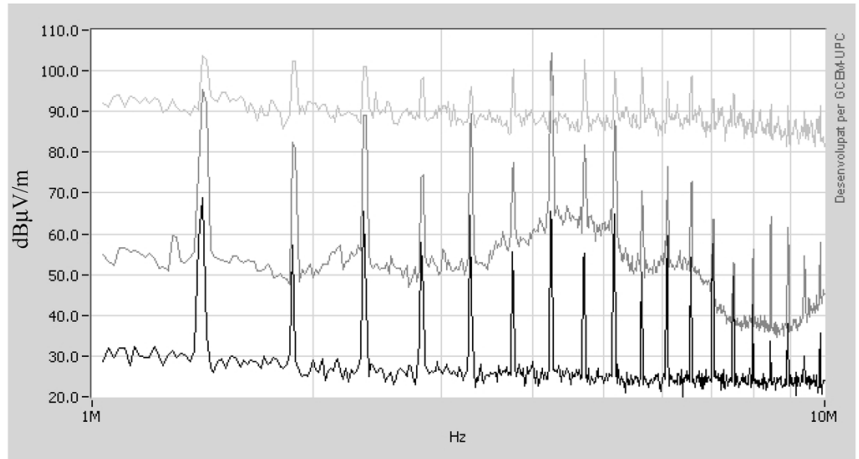

(b)

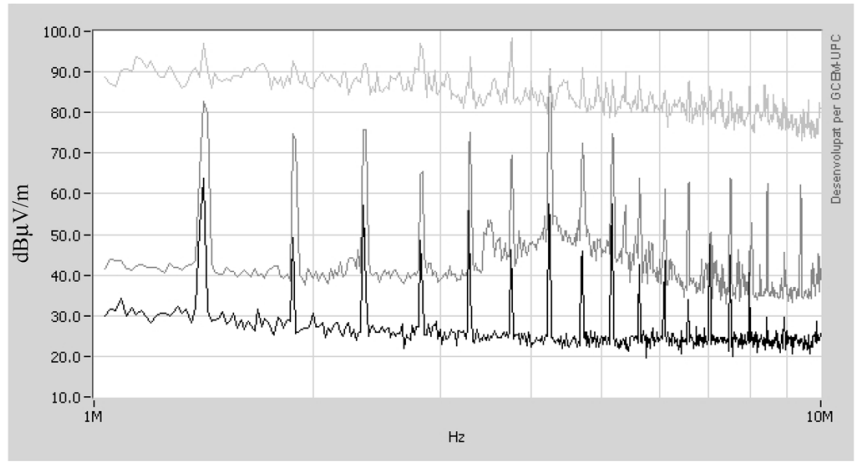

(c)

Fig. 2. Measurement results for a load resistor (black line), cutting a piece of meat (dark grey line) and with provoked arcing (light grey line) at $3 \mathrm{~m}$ from the EUT. (a) ROD antenna measurements. (b) Loop antenna perpendicular to the EUT. (c) Loop antenna parallel to the EUT.

situations: the first one with the ESU unit working with a resistor as a load, and the second when arcing with the proposed measurement setup (Fig. 3). Fig. 5 shows the results for the time domain electric field measurement. When an arc discharge is produced we observe that the measured electric field is up to 10 times higher than when there is no arc, and that the periodic behavior observed when there is no arc [Fig. 5(a)] is substituted by a broad-band signal [Fig. 5(b)].

With these kinds of broad-band/narrow-band emissions it is difficult to establish a general measurement setup or an adequate radiated emission limit to be considered. If we wish to assess the effect in an analogue communication system, the usual bandwidth to consider is $9 \mathrm{kHz}$, set by CISPR [14] at this EMI frequency band. Within these measurements conditions, the ESU emissions when arcing are actually about $40 \mathrm{~dB}$ greater than those for the resistor load. As the arcing effect is not easily generated in the EMC lab because of the generation procedure explained before, measurements could be made with a resistor, but taking into account that the real EMI disturbing those communication systems will be $40 \mathrm{~dB}$ greater. It is also necessary to mention that 


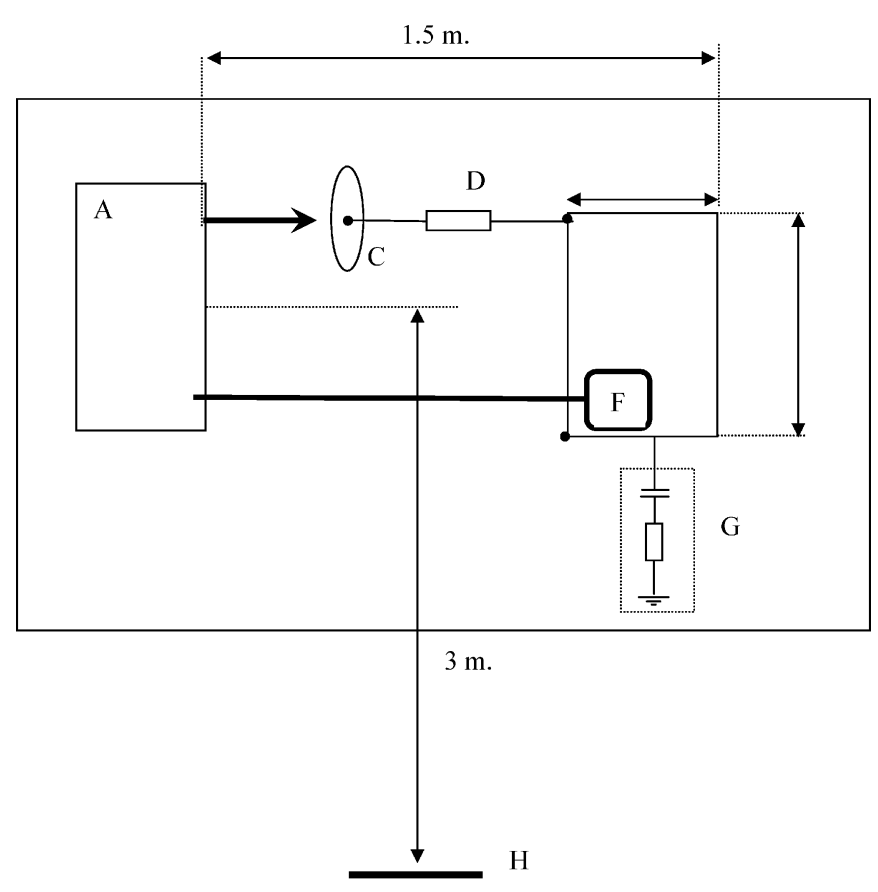

A: ESU

B: Active electrode

C: Metal plate for arcing

D: Patient resistance $500 \Omega 200 \mathrm{~W} \quad \mathrm{~L}<5 \mu \mathrm{H}$

E: Metal plate for patient simulation

F: Neutral electrode

G: patient to earth coupling $220 \mathrm{pF}, 510 \Omega$

H: antenna

Fig. 3. Measurement setup to consider the arcing phenomenon in the radiated EMI measurements.

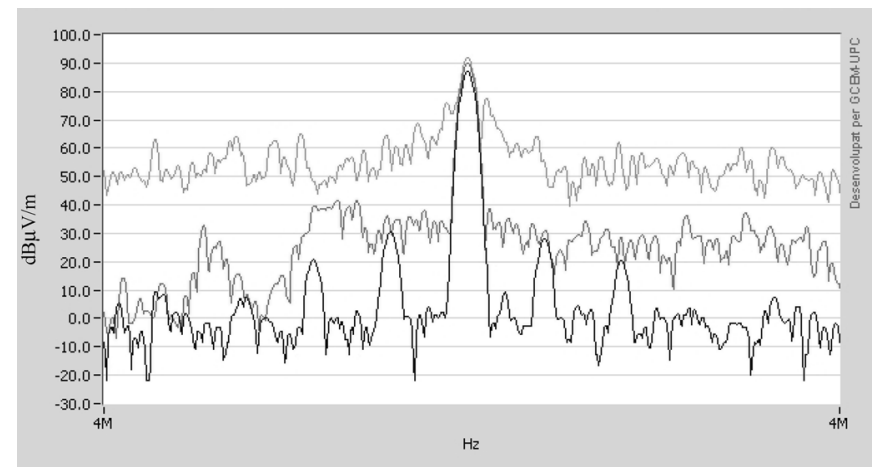

Fig. 4. Measurements results with a loop antenna perpendicular to measurement setup at $3 \mathrm{~m}$ performed with a $10-\mathrm{Hz}$ resolution bandwidth. Black line (bottom trace) with load resistor, dark grey line (central trace) cutting a piece of meat and light grey line (upper trace) with the proposed measurement setup for arcing.

Quasi Peak detector measurements will not reduce emissions because of the high repetition rate of the interference.

Nevertheless other systems such as digital communication systems or other noncommunication devices will provide very different responses to a mixture of broad-band and narrow-band interferences. It seems pointless to increase the immunity levels required for the all the susceptible devices by $40 \mathrm{~dB}$ without a detailed analysis. More useful information for designing the immunity behavior of near sensitivity devices could be given by showing the emissions from the ESU loaded with a resistor in the frequency domain, which is the narrow-band emission, and the arcing effect (broad-band) in the time domain.

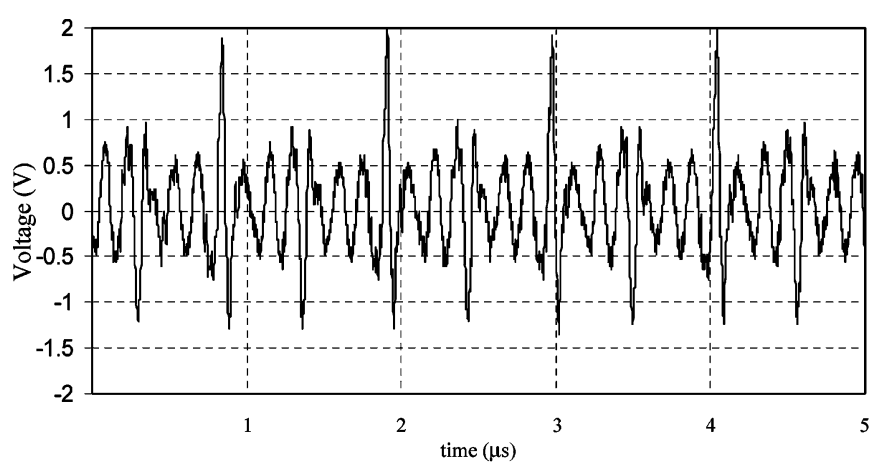

(a)

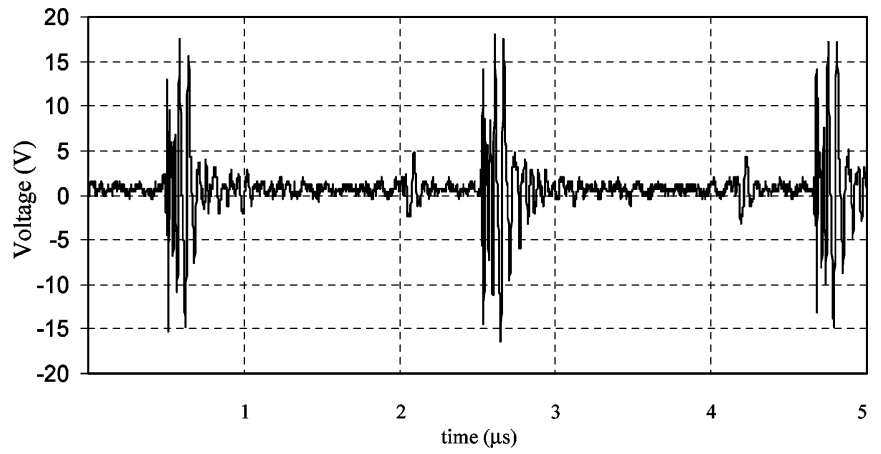

(b)

Fig. 5. Time domain electric field measurement (a) working with a resistor as a load and (b) when arcing.

\section{CONCLUSION}

Radiated electromagnetic disturbance from ESUs could lead to a medical system failure in operating rooms. When an ESU is used, large amounts of energy at hundreds of kilohertz are applied to the patient through cables which generate electric and magnetic radiated fields. Because other medical equipment is in close proximity, a near field EMC problem is likely to occur. In the low-power ESU analyzed in this paper, the electric field measured at a distance of $3 \mathrm{~m}$ reaches the $0.5 \mathrm{~V} / \mathrm{m}$ value.

Although near field radiated measurement is the more accurate testing method to evaluate the whole system performance, these types of measurements are not practical for characterizing ESUs at the EMC labs. Far field measurements with a setup devoted exclusively to this procedure are proposed instead. The ESU setup must take into account the effect of the patient as the electrical load, the surgeon handling the equipment and the arcing effect at cutting mode.

When we consider all these effects in real equipment use, such as cutting a piece of meat, the EMI radiated by an ESU shows a large increase in comparison with the setup proposed in the standards. Because the ESU narrow-band interference becomes broad-band when arcing occurs, a frequency and temporal domain characterization is proposed to correctly address the immunity of the sensitive medical equipment located near the ESU.

\section{REFERENCES}

[1] W. S. Boivin et al., "Measurement of electromagnetic field strengths in urban and suburban hospital operating rooms," in Proc. 19th Annu. Int. Conf. Engineering in Medicine and Biology Society, 1997, pp. 2539-2542.

[2] K.-S. Tan and I. Hinberg, "Measurement of electrical and magnetic fields from an electrosurgical device that interferes with other medical devices," in Proc. 15th Annu. Int. Conf. Engineering in Medicine and Biology Society, 1993, p. 1428. 
[3] H. Kawasaki, H. Egawa, M. Takasaki, and K. Yokoyama, "Malfunction of infusion pumps due to interference from an electrosurgical unit," Masui, vol. 40, no. 6, pp. 997-1002, Jun. 1991.

[4] F. Arambula Cosio et al., "EMC aspects of active robotic systems for surgery: the robotic prostatectomy experience," Med. Biol. Eng. Comput., pp. 436-440, 1997.

[5] R. Lafreniere et al., "Preparation of the operating room-Section 1: Basic surgical and preoperative considerations," in ACS Surgery: Principles and Practice. Danbury, CT: WebMD, Oct. 2003, pp. 1-14.

[6] J. L. Eggleston and W. W. von Maltzahn, "Electrosurgical devices," in The Biomedical Engineering HandBook, J. D. Bronzino, Ed., 2nd ed. Boca Raton, FL: CRC, 2000.

[7] B. Liljestran et al., "RF exposure during use of electrosurgical units," Electromagn. Biol. Med., vol. 22, pp. 127-132, Jan. 2003.

[8] Medical Electrical Equipment-Part 2-2: Particular Requirements for the Safety of High Frequency Surgical Equipment, ANSI Standard EN 60 601-2-2:2000, 1998.

[9] M. Fernández, M. Quílez, and F. Silva, "Near field EMI measurements in medical environments," in Proc. Electromagnetic Compatibility Symposium (EMC), Zurich, Switzerland, 2001, pp. 77-80.

[10] C. Gabriel, Compilation of the Dielectric Properties of Body Tissues at RF and Microwave Frequencies Brooks Air Force, Tech. Rep. AL/OE-TR-1996-0037.

[11] S. Gabriel, R. W. Lau, and C. Gabriel, "The dielectric properties of biological tissues: II. Measurements in the frequency range $10 \mathrm{~Hz}$ to 20 GHz," Phys. Med. Biol., vol. 41, no. 11, pp. 2251-2269.

[12] Medical Electrical Equipment-Part 2-25: Particular Requirements for the Safety of Electrocardiographs, ANSI Standard EN 606 012-25:1995.

[13] J. R. LaCourse, "The voltage-current characteristic of an electrosurgical arc," in Proc. 15th Annu. Int. Conf. the Engineering in Medicine and Biology Society, 1992, pp. 1136-1137.

[14] Specification for Radio Disturbance and Immunity Measuring Apparatus and Methods, ANSI Standard CISPR 16, Nov. 2003.

\section{On the Selection of Optimal Flip Angles for $T_{1}$ Mapping of Breast Tumors With Dynamic Contrast-Enhanced Magnetic Resonance Imaging}

\author{
Jeff L. Zhang* and T. S. Koh
}

\section{INTRODUCTION}

Recent studies have shown that the approach of dynamic contrastenhanced magnetic resonance imaging (DCE MRI) offers a promising tool for the differential diagnosis of breast tumors [1]-[3]. It involves the intravenous injection of a gadolinium $(\mathrm{Gd})$ contrast medium and rapid temporal imaging to monitor the changes in the MR signal as a function of time. The primary effect of Gd is the shortening of the spinlattice relaxation time $T_{1}$, resulting in greater signal in $T_{1}$-weighted images. For low concentrations $(C)$ of the contrast medium, the change in relaxation rate $R_{1}\left(=1 / T_{1}\right)$ is proportional to $C$, i.e.,

$$
R_{1}^{c}-R_{1} \propto C
$$

where $R_{1}^{\mathrm{c}}$ and $R_{1}$ are, respectively, the postcontrast and precontrast relaxation rates [4]. If the changes in $T_{1}$ values can be accurately measured as a function of time $(t)$, an important task in DCE MRI is to further deduce $C(t)$, from which physiological variables associated with tumor microcirculation can be estimated by tracer kinetic modeling [5].

A class of pulse sequences suitable for $T_{1}$-weighted DCE MRI is the spoiled gradient-recalled echo (SPGR) or fast low-angle shot (FLASH) sequences, which produces relatively artifact-free images with good temporal resolution [6], [7]. The relationship between the FLASH signal $S$ and the various imaging parameters can be given by [6]-[8]

$$
S=\frac{M_{0}\left[1-\exp \left(-\frac{T_{R}}{T_{1}}\right)\right] \sin \alpha}{1-\exp \left(-\frac{T_{R}}{T_{1}}\right) \cos \alpha}
$$

where $M_{0}$ is the equilibrium magnetization (which accounts for the proton density and machine gain), $T_{R}$ is the radio frequency (RF) repetition time, and $\alpha$ denotes the RF flip angle. Implicit in (2) is the assumption of $T_{E} \ll T_{2}^{*}$, where $T_{E}$ is the echo delay time, and $T_{2}^{*}$ is the spin-spin relaxation time. Rapid $T_{1}$ measurement can be achieved by acquiring at least two FLASH images at different RF flip angles, from which the two unknowns in (2) (i.e., $M_{0}$ and $T_{1}$ ) can be calculated. With $T_{\mathrm{R}}$ limited by temporal resolution, the selection of appropriate flip angles has the potential of improving the accuracy of the measured $T_{1}$ [6], [7]. The estimation of $T_{1}$ using this variable flip angle method was found to be in good agreement with the conventional saturation recovery method [6], [8].

In the following, we proposed a method for the selection of optimal angles for both precontrast and postcontrast $T_{1}$ mapping of breast tumors. Monte Carlo simulation studies were carried out to evaluate and compare the present approach with an existing approach.

\section{METHODS}

\section{A. Optimal Angles for Precontrast $T_{1}$}

Equation (2) can be re-cast into the linear form $Y=b X+a[6]$ as

$$
\frac{S}{\sin \alpha}=\exp \left(-\frac{T_{R}}{T_{1}}\right) \frac{S}{\tan \alpha}+M_{0}\left[1-\exp \left(-\frac{T_{R}}{T_{1}}\right)\right]
$$

and the problem of choosing $N$ optimal flip angles to most accurately determine $T_{1}$, would correspond to the optimal placement of $N$ points along the linear regression line, such that the error in $T_{1}$ is minimized.

Digital Object Identifier 10.1109/TBME.2006.873391 